Relations industrielles

Industrial Relations

\title{
Les partenaires sociaux face aux problèmes de productivité et d'emploi, par Yves Delamotte, Paris, O.C.D.E., 1971, 228 pp.
}

\section{Bertrand Belzile}

Volume 28, numéro 1, 1973

URI : https://id.erudit.org/iderudit/028378ar

DOI : https://doi.org/10.7202/028378ar

Aller au sommaire du numéro

Éditeur(s)

Département des relations industrielles de l'Université Laval

ISSN

0034-379X (imprimé)

1703-8138 (numérique)

Découvrir la revue

Citer ce compte rendu

Belzile, B. (1973). Compte rendu de [Les partenaires sociaux face aux problèmes de productivité et d'emploi, par Yves Delamotte, Paris, O.C.D.E., 1971, 228 pp.] Relations industrielles / Industrial Relations, 28(1), 213-214.

https://doi.org/10.7202/028378ar

Tous droits réservés @ C Département des relations industrielles de l'Université Laval, 1973
Ce document est protégé par la loi sur le droit d'auteur. L'utilisation des services d'Érudit (y compris la reproduction) est assujettie à sa politique d'utilisation que vous pouvez consulter en ligne.

https://apropos.erudit.org/fr/usagers/politique-dutilisation/ 
Dans l'ensemble, ce livre est de lecture agréable. Il peut être très avantageusement utilisé comme référence dans un cours de macro-économie, même élémentaire.

\section{Pierre FORTIN}

Les partenaires sociaux face aux problèmes de productivité et d'emploi, par Yves Delamotte, Paris, O.C.D.E., 1971, 228 pp.

L'auteur distingue les accords de productivité et les accords sur l'emploi, quoique les uns et les autres possèdent plusieurs similitudes. Les premiers sont intervenus en Grande-Bretagne et les seconds dans les pays continentaux. Dans les deux premiers chapitres, M. Delamotte traite des accords de productivité, et dans les chapitres 4,5 et 6 des accords sur l'emploi.

Par accord de productivité, l'auteur réfère ici à un accord par lequel les ouvriers acceptent de procéder à un ou plusieurs changements dans les pratiques du travail, changements qui mèneront eux-mêmes - en laissant de côté toute augmentation compensatrice des rémunérations - à une réduction du coût du travail ; et, en échange, l'employeur accepte un plus haut niveau des salaires ou d'autres avantages» (p. 24).

L'accord de Fawley, qui est intervenu en 1960 à la raffinerie du même nom, près de Southampton en Grande-Bretagne, illustre bien ce en quoi consistent les accords de productivité. Essentiellement, les employés y ont permis à la direction de modifier certaines règles d'utilisation de la main-d'œuvre et de répartition du travail, en échange d'augmentations du taux de base des salaires et d'autres avantages. Certaines de ces règles définissaient la structure de l'emploi et des salaires, d'autres concernaient l'encadrement immédiat des ouvriers, d'autres touchaient plus directement l'organisation et les pratiques du travail, d'autres enfin se rapportaient aux relations entre les corps de métier. En fait, les modifications apportées dans ces règles visaient l'accroissement de la productivité du travail (et du capital), d'où l'expression accords de productivité. De plus, ceux-ci formaient une entente globale (package deal).
Il faut s'empresser de noter que ces accords s'inscrivent dans une toile de fond législative afférente aux licenciements collectifs et plus généralement à l'adaptation aux changements technologiques. Ainsi, les droits particuliers qui sont reconnus aux travailleurs en vertu des accords de productivité s'ajoutent aux droits généraux qu'ils tiennent de la législation.

Au sens plus ou moins strict, on avait enregistré en Grande-Bretagne, en février $1969,2,500$ accords du genre. En effet, ils ne prévoyaient pas tous des changements dans les pratiques du travail et beaucoup d'entre eux se bornaient à prévoir une révision de la structure des salaires et des améliorations aux systèmes existants de rémunération fondés sur les résultats. Quoi qu'il en soit du contenu précis des accords particuliers, tous les accords de productivité visent à relever le niveau d'efficacité des entreprises. Il importe également de mentionner que les questions de sécurité d'emploi étaient traitées en priorité dans bon nombre des accords intervenus vers la fin des années 60 . Soulignons enfin que de tels accords n'ont pas tous été, comme celui de Fawley, négociés au niveau de l'établissement. Certains ont même été signés au niveau de toute une industrie.

D'après l'auteur, trois (3) circonstances peuvent expliquer le développement de tels accords: a) l'augmentation des heures supplémentaires; b) le développement chez les managers d'un esprit productivité ; et c) la politique gouvernementale en matière de productivité, de prix et de revenu. Quant aux implications des accords pour les managers et les syndicats, l'auteur en mentionne également trois principales. D'abord, la négociation d'un accord de productivité suppose des normes d'administration et de gestion des entreprises plus élevées, celles-ci en vue d'accroître la productivité du travail, non pas en demandant aux ouvriers de faire un plus grand effort, mais en tentant d'éliminer ce qui entretient dans les entreprises le sousemploi de la main-d'œuvre. Ensuite, les accords supposent une révision déchirante des conceptions prévalant traditionnellement en matière de " prérogatives de la direction ». Enfin, ils représentent une atteinte au principe de solidarité qu'inspire traditionnellement la politique syn- 
dicale en matière de salaires, par exemple, en introduisant une différenciation des rémunérations au sein d'une même branche d'activité, rendant ainsi moins applicable le principe corollaire de la comparabilité.

Les accords de production britanniques semblent avoir produit de très bons résultats: efficacité et rentabilité plus élevées; réduction du temps de travail; plus grande sécurité d'emploi face aux changements technologiques; justice et paix sociale accrues. La signification novatrice de tels accords est éclairée si on les replace dans l'ensemble du système britannique des relations industrielles, dont la pièce maîtresse réside encore dans les conventions collectives conclues pour toute une branche d'activité, système traditionnel à l'intérieur duquel s'est développé un système officieux de négociations d'entreprise, d'établissement, voire d'atelier.

\section{Accords continentaux sur l'emploi}

Tel que mentionné ci-dessus, ce sont des accords sur l'emploi qui existent sur le continent, et non des accords de productivité comme en Grande-Bretagne. L'auteur explique cette situation en prétendant que les derniers accords sont inutiles sur le continent. Et «ils sont inutiles parce qu'on ne trouve pas dans les entreprises de ces trois pays (Allemagne Fédérale, Suède et France) l'équivalent des règles ou pratiques qui peuvent faire obstacle dans des entreprises britanniques à une utilisation rationnelle de la main-d'œuvre, en introduisant des éléments de cloisonnement et de rigidité $\gg$ (p. 51). Une raison additionnelle, et peut être plus fondamentale que la précédente, qui peut aider à rendre compte de l'absence d'accords de productivité sur le continent, tient au type de relations professionnelles qui prévalent au plan des entreprises. Les accords dits sur l'emploi, dans les pays continentaux, semblent se distinguer des accords de productivité britanniques en ce sens qu'ils ne cherchent pas à faire accepter les changements (commandés par des considérations économiques ou techniques) en y liant les avantages particuliers sur le plan des rémunérations ou des horaires. Les accords sur l'emploi se bornent à poser un ensemble de règles qui garantissent que les changements à venir, et encore indéterminés, se feront avec moins d'inconvénients pour le personnel.

Ce qui précède rend compte de ce qui apparaît l'essentiel des deux types d'accords en question et rend compte aussi des soixante premières pages du volume. Les quelque cent (100) pages suivantes décrivent en détail les expériences qui ont eu lieu en Suède (un accord sur l'emploi affectant la productivité sans être un accord de productivité), en Allemagne Fédérale (des plans sociaux aux accords sur les conséquences sociales des mesures de rationalisation) et en France (les accords professionnels et interprofessionnels sur la sécurité d'emploi).

Dans les dix (10) dernières pages, l'auteur fait ressortir les caractéristiques essentielles des accords intervenus dans les quatre (4) pays déjà mentionnés et les commente eu égard à la législation, au plan des négociations collectives, à l'indemnisation des travailleurs déplacés, à la sécurité de l'emploi, à la mobilité professionnelle et géographique (et aux pratiques de travail) et enfin au cadre de la négociation.

Nous recommandons fortement la lecture de cet ouvrage. En plus d'être bien fait, il se rapporte à une question brûlante d'actualité.

\section{Bertrand B]ELZILE}

\section{Collective Bargaining by Public Em-} ployees in Canada : Five Models, by H.W. Arthurs, Ann Arbor, Michigan, Institute of Labor and Industrial Relations, The University of Michigan Wayne State University, $1971,166 \mathrm{pp}$.

This study is one of a series of parallel international comparative studies conceived and directed by Professors Russell Smith and Charles Rehmus of the University of Michigan. Professor Arthurs explored the Canadian scene while many other eminent scholars from other parts of the world provided an important contribution in describing the practices prevailing in their own country. Most of them were present at an international symposium on public sector labor relations held in New York City in May 1971. 